\section{Birth rate of millisecond pulsars}

SIR - The characteristic ages of millisecond pulsars are determined from their spin-down rates, but these might not be good estimates of their true ages. We recently reported ${ }^{1}$ a millisecond binary pulsar (J1012+5307) and tentatively identified the companion ( $\operatorname{star} \mathrm{X}$ ) as a white dwarf. Here we present optical colours and astrometry that confirm star $\mathrm{X}$ as the pulsar's companion, and that it is a white dwarf. The cooling age of the white dwarf is only $\sim 300$ million years, which is an order of magnitude less than the pulsar's characteristic age. If characteristic ages are generally in error by this amount, the birth rate of millisecond pulsars must be much greater than previously estimated.

The field containing PSR J1012+5307 and star X (see Fig. 2 of ref. 1) was

RADIO TIMING SOLUTION OF PSR J1012+5307

RA (J2000)

Dec. (J2000)

Period

Dispersion measure

Observed period derivative

Orbital period

Orbit semi-major axis

Orbit eccentricity

Epoch of period and

ascending node (MJD)

10 h $12 \min 33.4326(4) \mathrm{s}$

$53^{\circ} 07^{\prime} 02.66(1)^{\prime \prime}$

$0.00525574901198(2) \mathrm{s}$

$9.0207(4) \mathrm{cm}^{-3} \mathrm{pc}$

$1.46(8) \times 10^{-20}$

$0.604672713(5) \mathrm{d}$

$0.581816(6)$ light s

$<2 \times 10^{-5}$

49220.447499(1)

Numbers in parentheses represent twice the standard errors in the last quoted digit, inferred from a formal fit to the data.

observed on 21 October 1994 and 1 January 1995 during staff time at the Nordic Optical Telescape (NOT), on 22 January 1995 during service time on the Jacobus Kapteyn Telescope (JKT) and on 3 February 1995 during staff time at the Schmidt telescope at the Kvistaberg observatory. With $\sim 1$ arcsec seeing, we obtained 300 -s exposures in $\mathbf{B}, \mathrm{V}, \mathrm{R}$ and I filters on the NOT; $1,000 \mathrm{~s}$ in V, R and I on the JKT; and $3,600 \mathrm{~s}$ in $\mathrm{R}$ at the Schmidt telescope. We obtained photometry for star X from the NOT data by using the curve-of-growth technique ${ }^{2}$ in the PHOT procedure within the IRAF facility. We found that $V=$ $19.58 \pm 0.02, B-V=0.20 \pm 0.04, V-R=$ $0.09 \pm 0.03$ and $V-I=0.26 \pm 0.03$. Given the galactic coordinates of $\mathrm{J} 1012+5307$ $\left(l=160^{\circ}, b=51^{\circ}\right)$, any reddening effects on these data are negligible ${ }^{3}$.

1. Nicastro, L. et al. Mon. Not. R. astr. Soc. 273, L68-L70 (1995).

2. Stetson, P. B. Publs astr. Soc. Pacif. 102, 932-948 (1990).

3. Burstein, D. \& Heiles, C. Astr. J. 87, 1165-1189 (1982).

4. Lyne A. G. \& Smith, F. G. Nature 298, 825-827 (1982)

. Lyne, A. G. \& Smith, F. G. Nature 298, 825-827

6. Savonije, G. J. Nature 304, 422-423 (1983).

7. Iben, I. \& lutukov, A. V. Astrophys. J. 311, 742-752 (1986).

8. Camilo, F., Thorsett, S. E. \& Kulkarni, S. R. Astrophys. J. 421, L15-L18 (1994).

. Bell, J. F., Bailes, M. \& Bessell, M. S. Nature 364 603-605 (1993)

10. Bailyn, C. D. Astrophys. J. 411, L83-L85 (1993).

11. Danziger, I. J., Baade, D. \& Della Valle, M. Astr. Astrophys. 276, 382-388 (1993).

12. Lorimer, D. R. et al. Astrophys. J. 459, 933-938 (1995).
From the JKT and Schmidt data, using PPM guide-star positions, the position of star X at this epoch was found to be RA $(2000.0)=10 \mathrm{~h} 12 \mathrm{~min} 33.41 \mathrm{~s}$, dec. $(\mathrm{J} 2000.0)=53^{\circ} 07^{\prime} 02.7^{\prime \prime}$. Further radio observations of PSR J1012+5307 with the 76-m Lovell Telescope ${ }^{1}$ have resulted in significant improvements in the accuracy of the position of the pulsar, its period derivative and its orbital elements (see table). Together, these data show a position difference between PSR J1012+5307 and star $\mathrm{X}$ of only $0.2 \pm 0.5$ arcsec for epochs less than one year apart, making the identification almost certain, given the low stellar density in the field.

We obtain an upper limit of 33 mas $\mathrm{yr}^{-1}$ or the proper motion of star $\mathrm{X}$ from this new position and that reported in ref. 1 from the Palomar Sky Survey taken almost 40 years earlier. This implies a transverse speed $\leqslant 75 \mathrm{~km} \mathrm{~s}^{-1}$ at the distance, $d$, of $520 \mathrm{pc}$ inferred from the dispersion measure of $\mathrm{J} 1012+5307$. The pulsar shows strong interstellar scintillation, providing a rough estimate of the transverse speed of the binary system. Using standard analysis techniques ${ }^{4}$, we estimate this to be $\sim 50 \mathrm{~km} \mathrm{~s}^{-1}$, consistent with the upper limit for star X. Because a transverse speed, $v$, gives rise to an overestimate of the true period derivative ${ }^{5}$ of $\Delta \dot{P}=P v^{2} / c d$, we find that a better estimate of the intrinsic characteristic age $\tau=P / 2 \dot{P}$ of PSR $\mathrm{J} 1012+5307$ is 7 Gyr.

The $B-V, V-R$ and $V-I$ colours of star $\mathrm{X}$ are all consistent with a black-body temperature of $9,400 \pm 300 \mathrm{~K}$. Adopting the mass-radius relation reported in ref. 6 , we estimate the luminosity of a $0.15 M_{\odot}$ white dwarf at this temperature to be $L_{*} \approx$ $4.2 \times 10^{-3} L_{\odot}$. At a distance of $520 \mathrm{pc}$, this implies a visual magnitude of 19.3, in good agreement with the observed value. Thus both the colour and magnitude of star X are consistent with it being a $9,400-K$ white dwarf star.

Taking the luminosity $L_{*}=4.2 \times 10^{-3}$ $L_{\odot}$ from the cooling curve in ref. 7, we estimate the age of star X to be $\sim 300 \mathrm{Myr}-$ more than a factor of 20 below the characteristic age of J1012+5307. Given the strong evidence in favour of the association, and the relatively small separation of the binary pair $\left(\sim 0.3 R_{\odot}\right)$, the discrepancy between the observed and predicted luminosities might be due to the heating of star $\mathrm{X}$ by the spin-down energy loss of $\mathrm{J} 1012+5307$. However, a simple calculation shows that this makes only a minor contribution $\left(\sim 10^{-2} L_{*}\right)$ to the stellar luminosity. The discrepancy is surely due to the system being much younger than the pulsar characteristic age, implying that the initial spin period of $\mathrm{J} 1012+5307$ was similar to the presently observed value ${ }^{8}$. On much larger time-scales, similar, but less certain, discrepancies have been noted ${ }^{9-11}$ for the millisecond pulsar J0437-4715 and the anomalously high characteristic ages of some Galactic-disk millisecond pulsars which exceed the age of the Galaxy ${ }^{8}$.

This identification underlines the possible substantial overestimate of the ages of millisecond pulsars by use of their characteristic ages. If this is generally true, the birth rate required to sustain the observed Galactic population will be much greater than previous calculations ${ }^{12}$ have suggested. D. R. Lorimer, A. G. Lyne The University of Manchester, Nuffield Radio Astronomy Laboratories, Jodrell Bank, Macclesfield SK11 9DL, UK L. Festin

Astronomical Observatory,

Box 515, S-751 20, Sweden

\section{Nicastro}

Istituto di Tecnologie e Studie delle

Radiasioni Extraterrestri - CNR,

Via Gobetti 101, 40129 Bologna, Italy

\section{Localization of active zones}

SIR - Synaptic transmission is believed to proceed by the function of spatially distinct zones, which can be clearly identified by morphological studies ${ }^{1}$. This hypothesis, however, lacks direct functional evidence. Here we show that sites of generation of postsynaptic responses evoked by a single quantum of transmitter at the neuromuscular junction of frog are grouped in active zones with spatial parameters similar to those of morphologically determined active zones.

Extracellular miniature endplate potentials (e.m.e.p.ps) were simultaneously recorded using three independent electrodes placed perisynaptically ( $A$ in the figure). In controls we found that the amplitude of e.m.e.p.ps was reciprocal to the distance between the electrode and the site of synaptic current generation (active site $)^{2}$; thus, comparison of the amplitudes of unitary e.m.e.p.ps recorded by the three electrodes allowed us to determine the location of active sites in two dimensions.

Sites of spontaneous release were grouped in active zones dispersed across the nerve terminal ( $B$ in the figure; zones $a-f)$. The probability of localization of the active sites along the nerve terminal also revealed peaks corresponding to the active zones ( $C$ in the figure). The distance between the active zones was $1.3 \pm 0.5 \mathrm{~mm}$ while their length was $1.9 \pm 0.9 \mathrm{~mm}$ $(n=17)$, which corresponds well with those determined by morphological studies ${ }^{3,4}$. Some of the active zones did not extend 